\title{
Ultraviolet photo-ionisation in far-infrared selected sources
}

\author{
S. J. Curran ${ }^{1}$ and S. W. Duchesne ${ }^{2}$ \\ 1 School of Chemical and Physical Sciences, Victoria University of Wellington, PO Box 600, Wellington 6140, New Zealand \\ e-mail: Stephen. Curran@vuw.ac.nz \\ 2 International Centre for Radio Astronomy Research (ICRAR), Curtin University, Bentley, WA 6102, Australia
}

Received 3 May 2019 / Accepted 31 May 2019

\begin{abstract}
It has been reported that there is a deficit of stellar heated dust, as evident from the lack of far-infrared (FIR) emission, in sources within the Herschel-SPIRE sample with X-ray luminosities exceeding a critical value of $L_{\mathrm{X}} \sim 10^{37} \mathrm{~W}$. Such a scenario would be consistent with the suppression of star formation by the AGN, required by current theoretical models. Since absorption of the $21 \mathrm{~cm}$ transition of neutral hydrogen $\left(\mathrm{HI}_{\mathrm{I}}\right.$, which traces the star-forming reservoir, also exhibits a critical value in the ultraviolet band (above ionising photon rates of $Q \approx 3 \times 10^{56} \mathrm{~s}^{-1}$ ), we test the SPIRE sample for the incidence of the detection of $250 \mu \mathrm{m}$ emission with $Q$. The highest value at which FIR emission is detected above the SPIRE confusion limit is $Q=8.9 \times 10^{57} \mathrm{~s}^{-1}$, which is $\approx 30$ times that for the $\mathrm{H}_{\mathrm{I}}$, with no critical value apparent. Since complete ionisation of the neutral atomic gas is expected at $Q \gtrsim 3 \times 10^{56} \mathrm{~s}^{-1}$, this may suggest that much of the FIR must arise from heating of the dust by the AGN. However, integrating the ionising photon rate of each star over the initial mass function, we cannot rule out that the high observed ionising photon rates are due to a population of hot, massive stars.
\end{abstract}

Key words. galaxies: active - ultraviolet: galaxies - infrared: galaxies - X-rays: galaxies - galaxies: ISM - submillimeter: galaxies

\section{Introduction}

Feedback between active galactic nuclei (AGN) and their host galaxies is complex, with the central engine ionising the very material which feeds it (e.g. Di Matteo et al. 2005; Fabian 2012). The ionisation can arise from powerful outflows into the surrounding neutral medium (jet/radio-mode; e.g. Cano-Díaz et al. 2012; Farrah et al. 2012), as well as by ultraviolet (Silk \& Rees 1998) and X-ray (Fabian 1999) radiation emanating from the accretion disc surrounding the supermassive black hole (radiative/quasar-mode, Hardcastle et al. 2007; Heckman \& Best 2014). Both mechanisms suppress star formation through ionisation and heating of the neutral gas, this suppression being required by current theoretical models to reproduce the observed properties of active galaxies (e.g. Croton et al. 2006). Observational evidence of this has recently been claimed, where powerful AGN, as evident through their X-ray luminosity, lack $\lambda=250 \mu \mathrm{m}$ far-infrared (FIR) emission over the redshift range $1<z<3$. In the rest frame this corresponds to wavelengths of $63-125 \mu \mathrm{m}$, which trace the dust heated by stars (e.g. Dale \& Helou 2002), and so the absence of FIR emission is interpreted as the suppression of star formation in the X-ray luminous sources (Page et al. 2012).

Powerful AGN are also extremely bright in the UV, which is redshifted into the optical band at $z \gtrsim 3$, allowing quasistellar objects (QSOs) to be visible to ground-based telescopes over much of the observable Universe. Observational evidence of high UV luminosities rendering the star-forming material undetectable in the hosts of radio-loud QSOs (quasars) was suggested by the exclusive non-detection of the $21 \mathrm{~cm}$ transition of neutral hydrogen $\left(\mathrm{H}_{\mathrm{I}}\right)$ above a critical UV luminosity of $L_{\mathrm{UV}} \sim 10^{23} \mathrm{~W} \mathrm{~Hz}^{-1}$ (Curran et al. 2008). H I $21 \mathrm{~cm}$ absorption traces the cool, star forming, component of the gas and a subsequent model of a quasar placed within an exponential gas disc found that this luminosity, which corresponds to $Q \sim 3 \times 10^{56}$ ionising $(\lambda \leq 912 \AA)$ photons s ${ }^{-1}$, is just sufficient to ionise all of the neutral gas in the Milky Way (Curran \& Whiting 2012). Given that this is a large spiral galaxy, complete ionisation of the neutral gas would explain why $\mathrm{H}$ I has never yet been detected in sources with these UV luminosities (Curran et al. 2008, 2011, 2013a,b, 2016, 2017a,b, 2019; Allison et al. 2012; Grasha \& Darling 2011; Geréb et al. 2015; Aditya et al. 2016, 2017; Aditya \& Kanekar 2018; Curran \& Duchesne 2018; Grasha et al. 2019). Since $\mathrm{H}_{\text {I }} 21 \mathrm{~cm}$ absorption traces the reservoir for star formation, we may also expect the $250 \mu \mathrm{m}$ emission to be absent in the sources above the critical UV ionising photon rate, a possibility we investigate here.

\section{Analysis}

\subsection{Source matching}

The Herschel Multi-tiered Extragalactic Survey (HerMES, Oliver et al. 2012), utilised by Page et al. (2012), covers a $\sim 340 \mathrm{deg}^{2}$ field, operating at wavelengths of $\lambda=250,350$, and $500 \mu \mathrm{m}$ (Spectral and Photometric Imaging Receiver, SPIRE) and 100 and $160 \mu \mathrm{m}$ (Photodetector Array Camera and Spectrometer, PACS). We constructed a subset of extragalactic sources within the SPIRE dataset using the STARFINDER sourcefinder software (Diolaiti et al. 2000), with cross-identification being performed between the single-band catalogues (Roseboom et al. 2010), resulting in a merged catalogue of 340968 sources. We then searched the NASA/IPAC Extragalactic Database (NED) for sources within 6 arcsec of the SPIRE positions (as per Page et al. 2012, see Appendix A), which were crossmatched according to the minimum separation between sources, while ensuring no duplicate matches. After filtering out SPIRE sources without a counterpart nor a redshift, there were 62073 


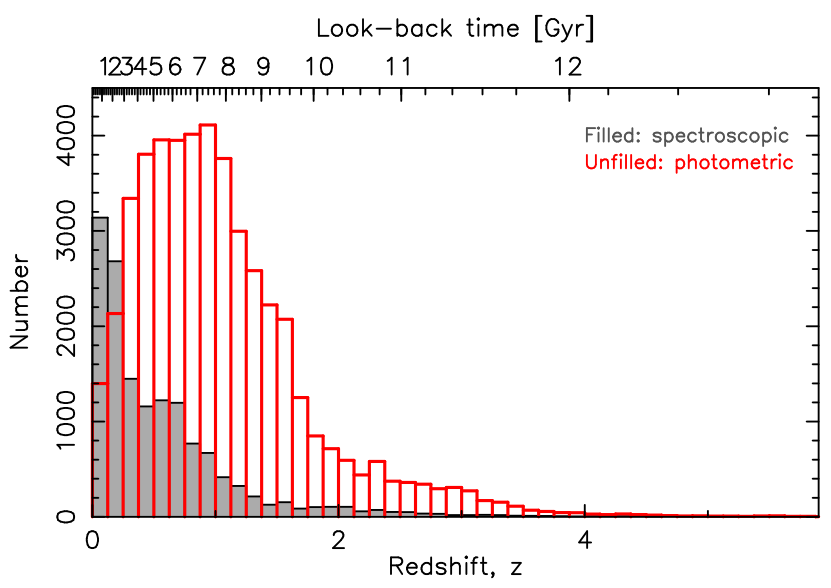

Fig. 1. Distribution of the spectroscopic (filled histogram) and the photometric redshifts (unfilled) for the 62073 matched SPIRE sources.

sources remaining. Of these, 14457 have spectroscopic redshifts (Fig. 1).

\subsection{Photometry fitting}

\subsubsection{Ultraviolet}

In order to obtain the UV luminosities, we queried NED, the Wide-Field Infrared Survey Explorer (WISE, Wright et al. 2010), the Two Micron All Sky Survey (2MASS, Skrutskie et al. 2006) and the Galaxy Evolution Explorer (GALEX data release GR6/7) ${ }^{1}$ databases. Each flux density measurement, $S_{v}$, was corrected for Galactic extinction (Schlegel et al. 1998) before being converted to a specific luminosity at the source-frame frequency via $L_{v}=4 \pi D_{\mathrm{L}}^{2} S_{v} /(z+1)$, where $D_{\mathrm{L}}$ is the luminosity distance to the source.

The ionising photon rate is defined as $Q \equiv \int_{v_{0}}^{\infty}\left(L_{v} / h v\right) \mathrm{d} v$ (Osterbrock 1989), where $v_{0}=3.29 \times 10^{15} \mathrm{~Hz}$ for the ionisation of neutral hydrogen. For the fitting, we require at least three $v \gtrsim 10^{15} \mathrm{~Hz}$ photometry points to which we fit a power law, giving $\log _{10} L_{v}=\alpha \log _{10} v+C \Rightarrow L_{v}=10^{C} v^{\alpha}$, where $\alpha$ is the spectral index and $C$ the intercept. This gives the ionising photon rate as

$Q=\frac{10^{C}}{h} \int_{v_{0}}^{\infty} v^{\alpha-1} \mathrm{~d} v=\frac{10^{C}}{\alpha h}\left[v^{\alpha}\right]_{v_{0}}^{\infty}=\frac{-10^{C}}{\alpha h} v_{0}^{\alpha}$,

where $\alpha<0$.

\subsubsection{Far-infrared}

Since we were also interested in the effect of the UV continuum on the dust temperature, we added the photometric data of the three SPIRE bands to that above, where we only included the data detected at $S_{v}>3 \sigma_{\text {conf }}$, where $\sigma_{\text {conf }}=6 \mathrm{mJy}$ is the SPIRE confusion limit (Nguyen et al. 2010; Smith et al. 2012). For $T_{\text {dust }} \lesssim h v / k$ (Younger et al. 2009), where $h$ is the Planck constant and $k$ the Boltzmann constant, we fitted a modified blackbody (Fig. 2) spectrum of the form

$S_{v} \propto \frac{v^{3+\beta}}{e^{h v / k T_{\text {dust }}}-1}$,

where $\beta$ is the spectral emissivity index (e.g. Casey 2012).

\footnotetext{
1 http://galex.stsci.edu/GR6/\#mission
}

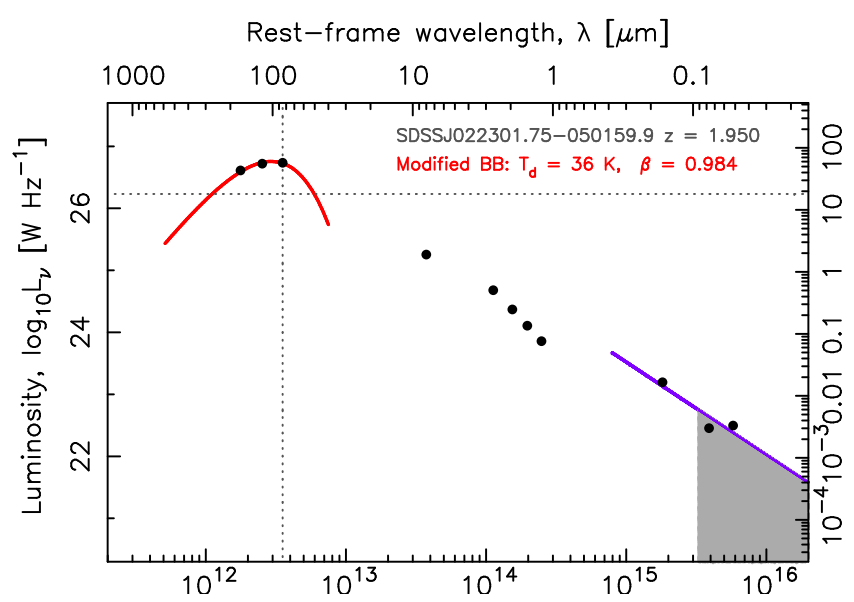

Fig. 2. Example of the FIR and UV photometry fits. The vertical line shows the observed-frame $250 \mu \mathrm{m}$ frequency and the horizontal line the SPIRE confusion limit of $3 \sigma_{\text {conf }}=18 \mathrm{mJy}$. The shaded region shows the $\lambda \leq 912 \AA$ band over which the ionising photon rate is derived.

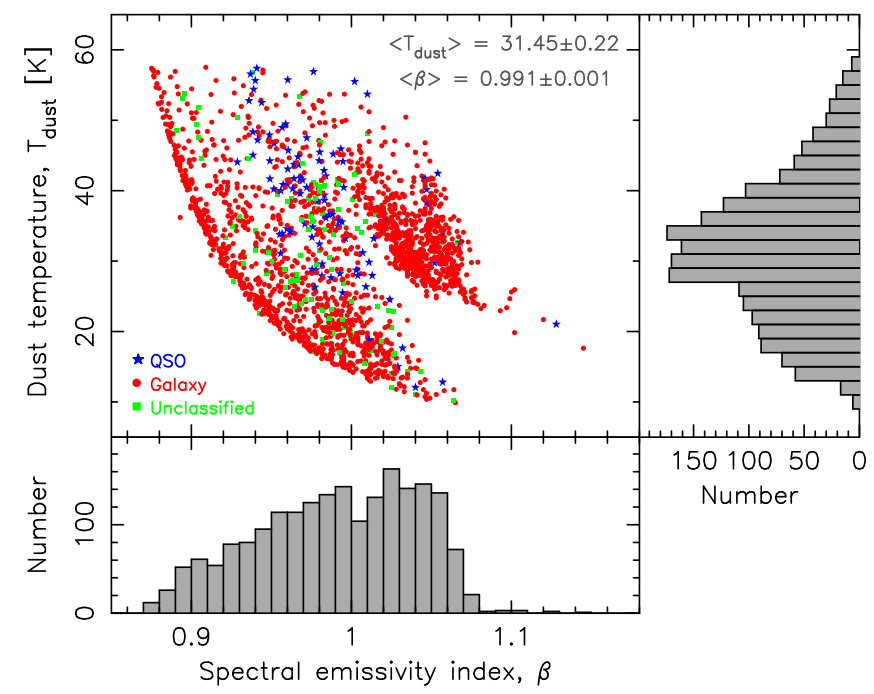

Fig. 3. Distribution of spectral emissivity index and dust temperature, where $T_{\text {dust }} \leq 1.2 \times 10^{12}(z+1) h / k$, giving a sample of $n=2013$. The shapes represent the NED classification: stars for QSOs, circles for galaxies, and squares for unclassified.

For this, we used a non-linear least-squares fitting of $\beta$ and $T_{\text {dust }}$ simultaneously, giving the distribution shown in Fig. 3. This yields a mean dust temperature of $\left\langle T_{\text {dust }}\right\rangle=31 \mathrm{~K}$ and a mean spectral emissivity index of $\langle\beta\rangle=0.99$. This is considerably lower than the canonical $\beta=1.5$ (Blain et al. 2003; Younger et al. 2009 and references therein), although this was used as the initial estimate. Forcing $\beta=1.5$ could not satisfactorily fit the data, although we do note that $\beta \approx 1.0$ may not be unexpected (Hildebrand 1983).

\section{Effects of the ultraviolet continuum}

\subsection{Critical ionising photon rate}

As stated in the introduction, our main motivation was to test whether the critical ionising photon rate found for $\mathrm{H}_{\mathrm{I}} 21 \mathrm{~cm}$ absorption also applied to FIR emission, where an apparent critical $\mathrm{X}$-ray luminosity may suggest the suppression of star formation. The highest ionising photon rate at which $21 \mathrm{~cm}$ absorption has 


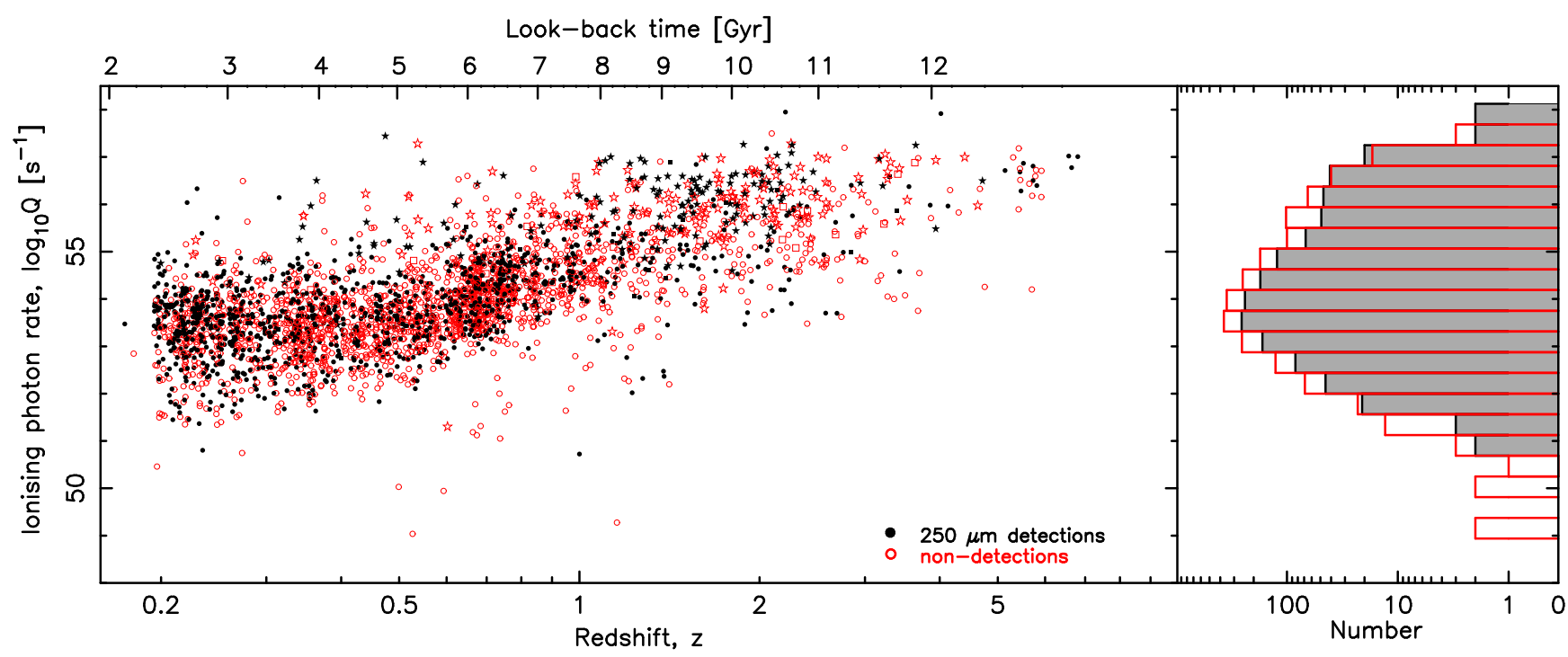

Fig. 4. Ionising $(\lambda \leq 912 \AA)$ photon rate vs. redshift for the sources with spectroscopic redshifts. The filled symbols and histogram represent the FIR detections, unfilled for the non-detections. As in Fig. 3, the shapes represent the NED classification: stars for QSOs, circles for galaxies, and squares for unclassified.

been detected is $Q=2.5 \times 10^{56} \mathrm{~s}^{-1}$, above which there are 87 nondetections, which is significant at $5.66 \sigma$ (Curran et al. 2019). Since $21 \mathrm{~cm}$ absorption traces the cool, neutral gas that fuels the star formation, we may therefore expect a similar critical rate if the FIR emission is dominated primarily by stellar activity.

Of the sample, there is sufficient UV photometry for 3315 sources, of which 1347 are considered $250 \mu \mathrm{m}$ detections (where $S_{250 \mu \mathrm{m}}>18 \mathrm{mJy}$, Sect. 2.2.2), giving a detection rate of $40.6 \%$. As Fig. 4 shows, unlike for $21 \mathrm{~cm}$ absorption, the detections and non-detections share the same range of ionising photon rates, with $250 \mu \mathrm{m}$ emission being detected up to $Q=8.9 \times 10^{57} \mathrm{~s}^{-1}$. This is considerably higher than that observed for $\mathrm{H}_{\mathrm{I}} 21 \mathrm{~cm}$ absorption and the model prediction that all of the neutral gas in a large galaxy is ionised at $Q \gtrsim 3 \times 10^{56} \mathrm{~s}^{-1}$ (Curran $\&$ Whiting 2012). Thus, unlike the X-ray emission (Page et al. 2012, but see Appendix A), we see no distinction between the distribution of FIR detections and non-detections at high ionising photon rates.

\subsection{Dust heating}

Interstellar dust has an absorption cross-section that peaks at ultraviolet wavelengths, thus making the re-emitted FIR radiation a sensitive tracer of dust heating. From the modified blackbody fits to the FIR photometry, we can also investigate heating of the dust by the ultraviolet emission. Of the $250 \mu$ m detections, there are 404 sources to which we could fit a modified blackbody and which have sufficient UV photometry (e.g. Fig. 2). These exhibit a strong correlation between the dust temperature and the ionising photon rate (Fig. 5), with a generalised non-parametric Kendall-tau test giving a probability of $P(\tau)=5.28 \times 10^{-12}$ for the $T_{\text {dust }}-Q$ correlation arising by chance, which is significant at $S(\tau)=6.90 \sigma$ assuming Gaussian statistics. However, due to the flux limitation introduced by the SPIRE confusion limit, there will be a bias towards the most FIR luminous, and thus most UV luminous, sources with increasing redshift. Given that for a modified blackbody, the temperature and intensity are not independent (Sect. 2.2.2), this will also lead to an apparent increase in the dust temperature.

In order to correct for this, we obtained the integrated intensity over 40-1000 $\mu \mathrm{m}$ (Yang et al. 2007, cf. Fig. 2) from

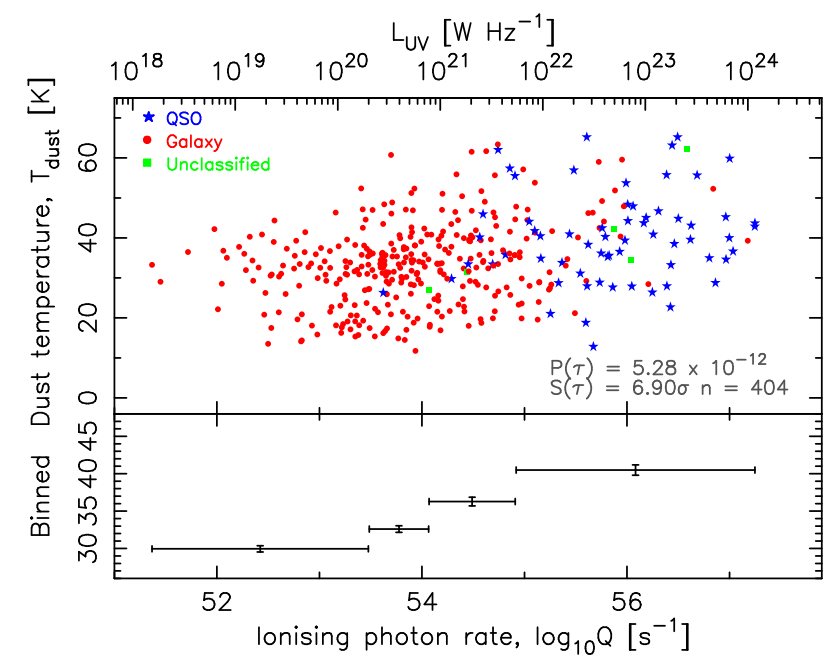

Fig. 5. Dust temperature vs. ionising photon rate. Bottom panel: data are shown in equally sized bins. The horizontal bars show the range of points in the bin and the vertical error bars the $1 \sigma$ uncertainty in the mean value.

$I_{\mathrm{FIR}}=\int_{3.0 \times 10^{11} \mathrm{~Hz}}^{7.5 \times 10^{12} \mathrm{~Hz}} \frac{2 \pi h v^{3+\beta}}{c^{2}} \frac{1}{e^{h v / k T_{\text {dust }}}-1} \mathrm{~d} v$,

from which a specific intensity $\left(I_{250 \mu \mathrm{m}}\right)$ is compared to the corresponding specific luminosity $\left(L_{250 \mu \mathrm{m}}\right.$, e.g. Fig. 2$)$ in order to provide the scaling to $L_{\mathrm{FIR}}$ (Fig. 6). From this we see that many of the luminosities are in excess of $L_{\mathrm{FIR}} \gtrsim 10^{12} L_{\odot}$, qualifying the sources as Ultra-Luminous Infrared Galaxies (ULIRGs). Normalising the ionising photon rate by the FIR luminosity (Fig. 7), the correlation disappears. This and Fig. 6 therefore indicate that the increase in dust temperature with redshift ${ }^{2}$ is due to the flux

2 For example, $T_{\text {dust }}=19-25 \mathrm{~K}$ in nearby galaxies (Galametz et al. $2012), T_{\text {dust }}=31-36 \mathrm{~K}$ in intermediate redshift starburst galaxies and AGN (Kovács et al. 2010; Magdis et al. 2013) and $T_{\text {dust }} \approx 40 \mathrm{~K}$ in intermediate and high redshift ULIRGs (Yang et al. 2007 and Younger et al. 2009 , respectively). 


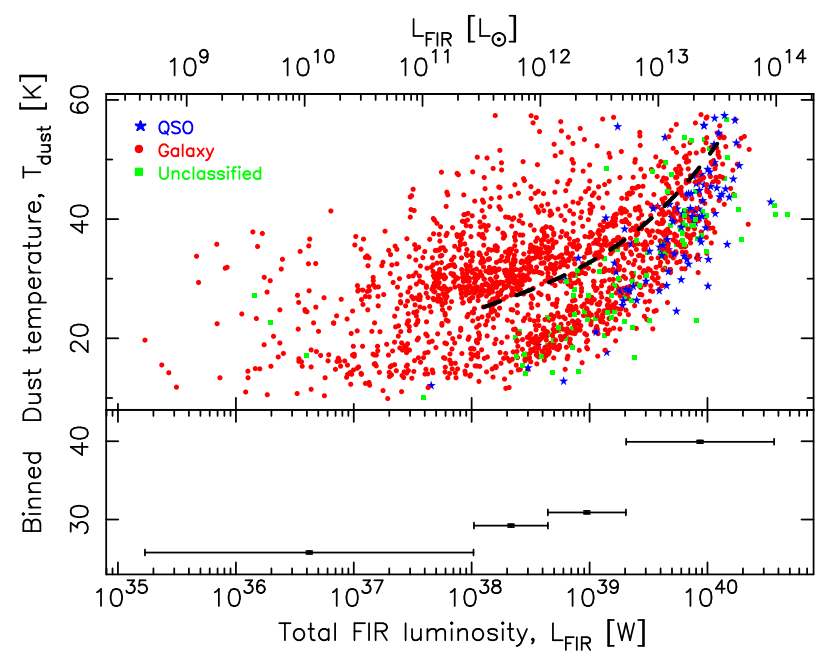

Fig. 6. Dust temperature vs. total FIR luminosity. The dashed curve shows the fit for the SPIRE-mm sources (Roseboom et al. 2012).

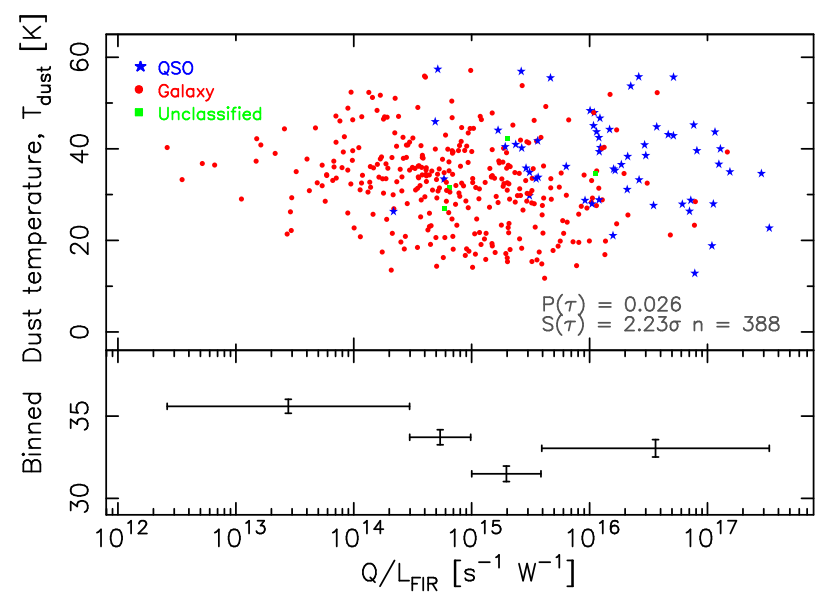

Fig. 7. Dust temperature vs. the ionising photon rate normalised by the total FIR luminosity.

limitation introducing a bias towards the most FIR (and UV) luminous sources.

\subsection{Stellar versus AGN activity}

The FIR emission is believed to be dominated by stellar heating (Rieke \& Lebofsky 1979 and references therein), whereas the mid-infrared (MIR, $\lambda=24 \mu \mathrm{m}$ ) emission can arise in photon dominated or HII regions (e.g. Galliano et al. 2018), and from AGN heated dust in the circumnuclear torus (Hatziminaoglou et al. 2010) ${ }^{3}$. However, the absence of the same critical ionising photon rate, which completely ionises the neutral atomic gas, and thus suppresses the star formation, at $Q \gtrsim 3 \times 10^{56} \mathrm{~s}^{-1}$ (Curran \& Whiting 2012), may suggest that much of the FIR emission also arises from dust heated by the AGN (e.g. de Grijp et al. 1985; Curran et al. 2001; Nardini et al. 2010).

For a blackbody with $T=5770 \mathrm{~K}$ and a radius of $R_{\odot}=696 \times$ $10^{8} \mathrm{~m}$, the ionising photon rate of the Sun is $Q_{\odot}=7.9 \times 10^{35} \mathrm{~s}^{-1}$. Thus, UV luminosities corresponding to $Q \gtrsim 10^{57} \mathrm{~s}^{-1}$ (Fig. 4) would require $\gtrsim 10^{21}$ solar mass stars and so, while an older (lower mass) population of stars could contribute significantly

\footnotetext{
3 Invoked by unified schemes of AGN (e.g. Antonucci 1993; Urry \& Padovani 1995).
}

Table 1. Estimated properties of stars of various temperatures.

\begin{tabular}{lccc}
\hline \hline$T_{\star}[\mathrm{K}]$ & 20000 & 35000 & 50000 \\
\hline$L_{\text {total }}\left[L_{\odot}\right]$ & 4200 & $2 \times 10^{5}$ & $2 \times 10^{6}$ \\
Radius $\left[R_{\odot}\right]$ & 5.4 & 11 & 17 \\
Mass $\left[M_{\odot}\right]$ & 11 & 31 & 60 \\
$t_{\mathrm{MS}}[\mathrm{yr}]$ & $3 \times 10^{7}$ & $2 \times 10^{6}$ & $4 \times 10^{5}$ \\
$Q_{\star}\left[\mathrm{s}^{-1}\right]$ & $2.7 \times 10^{46}$ & $1.9 \times 10^{48}$ & $3.4 \times 10^{49}$ \\
$N_{\text {stars }}$ & $7.9 \times 10^{8}$ & $7.0 \times 10^{7}$ & $1.5 \times 10^{7}$ \\
$\mathrm{SFR}\left[M_{\odot} \mathrm{yr}^{-1}\right]$ & 300 & 1100 & 2500 \\
\hline
\end{tabular}

Notes. $L_{\star}$ is the stellar luminosity estimated from the main sequence, followed by the radius required to equate this to the bolometric intensity obtained from the Planck function. The stellar mass is estimated from the mass-luminosity relation, $L_{\star} / L_{\odot}=\left(M_{\star} / M_{\odot}\right)^{3.5}$, and the main sequence lifetime, $t_{\mathrm{MS}}$, from $t_{\mathrm{MS}} \propto 1 / M^{2.5}$. $N_{\text {stars }}$ is the number of stars of this mass according to the Salpeter initial mass function, normalised to a total stellar mass of $3 \times 10^{12} M_{\odot}$, followed by the star formation required to maintain this (see main text).

to the FIR luminosity (Calzetti et al. 2010; Groves et al. 2012; Li et al. 2013), they cannot account for the UV luminosity. In order to explore the stellar masses required, the ionising photon rate of a star is obtained from the integrated intensity, $I_{v}$, via

$Q_{\star} h=L_{\mathrm{UV}}=4 \pi R_{\star}^{2} \int_{v_{0}}^{\infty} I_{\nu} \mathrm{d} v$,

where $I_{v}=\frac{2 \pi h v^{3}}{c^{2}} \frac{1}{e^{h v / k T_{\star}}-1}$,

$v_{0}=3.29 \times 10^{15} \mathrm{~Hz}$ corresponds to $\lambda=912 \AA$, where the hydrogen is ionised, and $T_{\star}$ is the surface temperature of the star. To obtain the luminosity we require a value for the surface area of the star $\left(4 \pi R_{\star}^{2}\right)$, which we estimate from the comparison of the bolometric intensity, $I=\int_{0}^{\infty} I_{\nu} \mathrm{d} v$, with the bolometric luminosity obtained from the main sequence using $\log _{10} L_{\star}=$ $6.50 \log _{10} T_{\star}-24.37$. Examples of the derived properties are given in Table 1.

In order to obtain the total ionising luminosity, we integrate both the initial mass function (IMF or $\xi$ ) and $Q_{\star}$ over the whole mass range, which we normalise to the approximate stellar mass of the Milky Way (Fig. 8). The total stellar mass is given by $M_{\text {total }}=\int_{M_{1}}^{M_{2}} M \xi \mathrm{d} M$ and it is dominated by the low mass stars, whereas the total ionising photon rate is dominated by the massive stars (see Table 1). If we truncate the IMF at $M_{\star}=25 M_{\odot}$ $\left(T_{\star} \approx 30000 \mathrm{~K}, t_{\mathrm{MS}} \approx 3 \times 10^{6} \mathrm{yr}\right)$ for the maximum possible mass, we obtain $Q_{\text {total }}=3 \times 10^{56} \mathrm{~s}^{-1}$, whereas setting this to $M_{\star}=100 M_{\odot}\left(T_{\star} \approx 65000 \mathrm{~K}, t_{\mathrm{MS}} \approx 1 \times 10^{5} \mathrm{yr}\right)$ gives $Q_{\text {total }}=7 \times 10^{57} \mathrm{~s}^{-1}$.

Thus, the total ionising photon rate from the stellar population within the source is very much dependent upon the upper end of the stellar mass distribution. Assuming no significant AGN contribution, the highest FIR luminosities of the sample indicate star formation rates of $\sim 10^{4} M_{\odot} \mathrm{yr}^{-1}$ (e.g. Kennicutt 1998), which we can use to constrain the upper mass end. In this instance, integrating over all star formation rates ${ }^{4}$, a total of $\mathrm{SFR}_{\text {total }}=1 \times 10^{4} M_{\odot} \mathrm{yr}^{-1}$ is reached for a maximum stellar

4 The specific star formation rate at a given mass is estimated from $\mathrm{SFR}=N_{\text {stars }} M / t_{\mathrm{MS}}$. For example, from Table 1 , we expect $8 \times 10^{8}$ stars of $\approx 10 M_{\odot}$, which have a lifetime of $t_{\mathrm{MS}} \approx 3 \times 10^{7} \mathrm{yr}$. This therefore requires $\mathrm{SFR} \approx 8 \times 10^{8} \times 10 / 3 \times 10^{7} \approx 300 M_{\odot} \mathrm{yr}^{-1}$ to maintain the observed luminosity. 


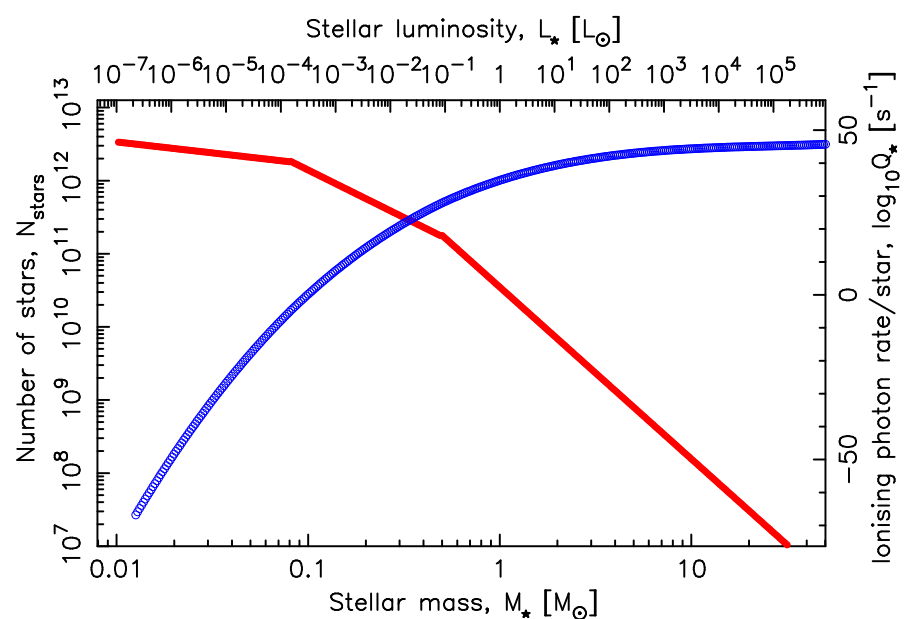

Fig. 8. Frequency of various stellar masses obtained from the initial mass function, $\xi=\xi_{0} M^{\alpha}$, normalised to give a total stellar mass of $M_{\text {total }}=4 \times 10^{11} M_{\odot}, \alpha=-0.3$ for $0.01<M_{\odot}<0.08, \alpha=-1.3$ for $0.08<M_{\odot}<0.5$, and $\alpha=-2.35$ for $M_{\odot}>0.5$ (Salpeter 1955; Kroupa 2001). The curve (right-hand scale) shows the approximate ionising photon rate per star.

mass of $M_{\star} \approx 40 M_{\odot}$, which gives $Q_{\text {total }}=9 \times 10^{56} \mathrm{~s}^{-1}$. However, even at relatively large look-back times, e.g. $\sim 10 \mathrm{Gyr}(z \sim 2)$, this implies a total stellar mass of $\sim 10^{13} M_{\odot}$, for a constant star formation rate over these first $\sim 3 \mathrm{Gyr}$. In addition to the total stellar mass, a steeper IMF would affect the stellar contribution to $Q_{\text {total }}$, for instance, $\alpha=-2.65$ for $1<M_{\odot}<10$ stars (Bastian et al. 2010), $\alpha=-5$ for $25<M_{\odot}<120$ stars in the Magellanic Clouds (Massey 2002), or $\alpha=-2.45$ to -2.85 in external galaxies (Úbeda et al. 2007; Bruzzese et al. 2015; Weisz et al. 2015). We therefore show the total ionising photon for a range of total masses and IMF indices in Table 2.

Since the total stellar mass is dominated by the more numerous, least massive, cooler stars, ionising photon rates above the critical $21 \mathrm{~cm}$ value favour $M_{\text {total }} \lesssim 4 \times 10^{11} M_{\odot}$, although it should be borne in mind that $\mathrm{SFR}_{\text {total }}=1 \times 10^{4} M_{\odot} \mathrm{yr}^{-1}$ is most likely an upper limit, due to an AGN contribution to the FIR luminosity (Morić et al. 2010; Nardini et al. 2010). For example, for a $50 \%$ contribution, the critical ionising photon rate is reached by just two of the canonical Galactic models $\left(M_{\text {total }}=4 \times 10^{11} M_{\odot}\right.$, Table 3$)$. This still begs the question of how the continual star formation is fuelled, although the stellar contribution to $Q_{\text {total }}$ will decrease further with an increasing AGN contribution. Furthermore, we have not accounted for shielding by dust nor how much this attenuates the observed UV flux. Also, while the critical $Q_{\text {total }}=3 \times 10^{56} \mathrm{~s}^{-1}$ is sufficient to ionise all of the neutral atomic gas in the Milky Way (where $n \lesssim 10 \mathrm{~cm}^{-3}$, Kalberla \& Kerp 2009), since $Q \propto n^{2}$ (Osterbrock 1989), denser gas (e.g. in molecular clouds where $n \gtrsim 10^{3} \mathrm{~cm}^{-3}$ ) requires much higher ionising photon rates (Roos et al. 2015). Simulations of a stellar population distributed within a galactic disc would be required in order to determine whether a distribution of UV luminous point sources would result in a Strömgren sphere of infinite radius, as is the case for a single centrally located $Q \gtrsim 10^{56} \mathrm{~s}^{-1}$ ionising source (Curran \& Whiting 2012).

Lastly, as Fig. 6 shows, many of the highest FIR luminous sources are classified as QSOs, and so are known to host a powerful AGN. Although they form only a small fraction (5\%) of the sources for which the FIR luminosity can be derived (Fig. 9), we see that the mean luminosity for the QSOs is an order

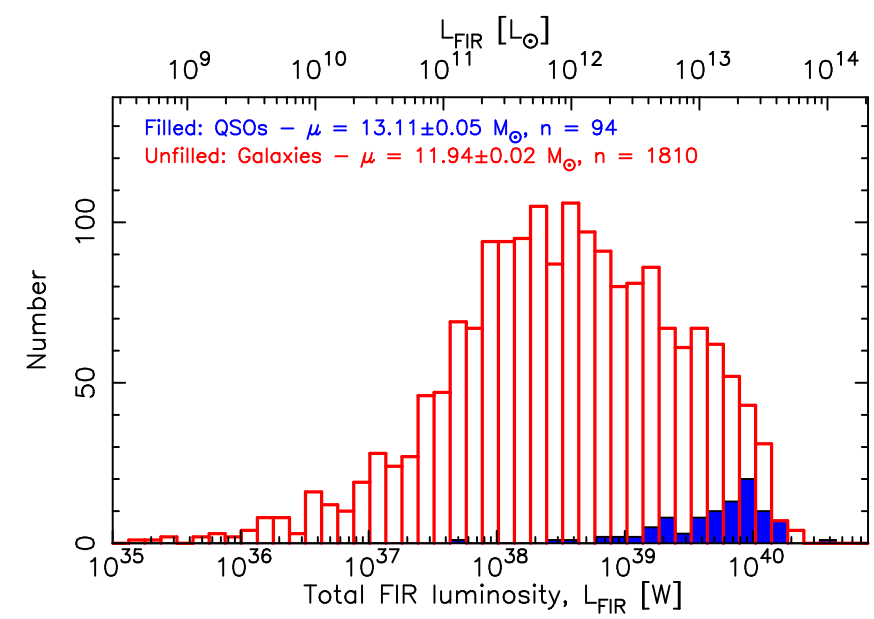

Fig. 9. Far-IR luminosity distribution of the known QSOs and galaxies of the sample.

of magnitude higher than for the galaxies (as is also the case for the UV, Fig. 5), lending support to the argument of an AGN contribution to the FIR emission in the most luminous sources. There does remain, however, a number of galaxies with these same luminosities.

\section{Conclusions}

For the past decade evidence has been building of a critical photo-ionisation rate in the ultraviolet band, above which all of the gas in the host galaxy is ionised. All of the observational evidence comes from searches of $\mathrm{H}_{\mathrm{I}} 21 \mathrm{~cm}$ absorption in redshifted radio sources, which has never been detected above rates of $Q=2.5 \times 10^{56} \mathrm{~s}^{-1}\left(L_{\mathrm{UV}} \gtrsim 10^{23} \mathrm{~W} \mathrm{~Hz}^{-1}\right.$, Curran et al. 2008, 2019). This observational result is supported by a model of a quasar placed within an exponential gas disc, for which $Q \gtrsim 3 \times 10^{56} \mathrm{~s}^{-1}$ is sufficient to ionise all of the neutral atomic gas in a large spiral (Curran \& Whiting 2012). However, current $21 \mathrm{~cm}$ absorption searches are insensitive to column densities of $N_{\mathrm{HI}} \ll 10^{20}$ atoms $\mathrm{cm}^{-2}$, and so the observations cannot rule out that the gas is merely heated or partially ionised to below the detection limit. Furthermore, the quasars with $L_{\mathrm{UV}} \gtrsim 10^{23} \mathrm{~W} \mathrm{~Hz}^{-1}$, tend to be type 1 objects, implying a direct view to the naked AGN, whereas at $L_{\mathrm{UV}} \lesssim 10^{23} \mathrm{~W} \mathrm{~Hz}^{-1}$, both type 1 (unobscured) and type 2 (obscured AGN) objects exhibit a $50 \%$ detection rate for $21 \mathrm{~cm}$ absorption (Curran \& Whiting 2010). This would suggest that the non-detection of H I above the critical UV luminosity is primarily an orientation effect, although it would mean that the low luminosity type 1 AGN are somehow different from their high luminosity counterparts.

Thus, it is of great interest to confirm the possibility of a critical ionising luminosity in another band. Page et al. (2012) report a critical X-ray luminosity $\left(L_{\mathrm{X}} \sim 10^{37} \mathrm{~W}\right)$ above which $250 \mu \mathrm{m}$ emission is not detected in $1<z<3$ SPIRE sources, leading to the conclusion that the star formation is suppressed in these objects. Since $21 \mathrm{~cm}$ absorption traces the reservoir for star formation, if the FIR emission is due mainly to stellar heated dust, we may also expect a critical UV luminosity above which the $250 \mu \mathrm{m}$ emission is suppressed. By matching the SPIRE sources to their NED counterparts, we obtain 14457 extragalactic objects with spectroscopic redshifts. Of these, there is sufficient UV photometry to determine the ionising photon rate for 3315 , and of the FIR detections (i.e. detected 
Table 2. Total ionising photon rates, $Q_{\text {total }}$, for various stellar mass distributions.

\begin{tabular}{|c|c|c|c|c|c|c|c|c|c|}
\hline \multirow{2}{*}{$\begin{array}{l}M_{\text {total }}\left[M_{\odot}\right] \\
\text { IMF index }\end{array}$} & \multicolumn{3}{|c|}{$4 \times 10^{11}$} & \multicolumn{3}{|c|}{$4 \times 10^{12}$} & \multicolumn{3}{|c|}{$4 \times 10^{13}$} \\
\hline & $\alpha=-2.35$ & $\alpha=-2.60$ & $\alpha=-2.85$ & $\alpha=-2.35$ & $\alpha=-2.60$ & $\alpha=-2.85$ & $\alpha=-2.35$ & $\alpha=-2.60$ & $\alpha=-2.85$ \\
\hline$M_{\max }\left[M_{\odot}\right]$ & 43 & 110 & 550 & 6 & 9 & 17 & 1 & 1 & 1 \\
\hline$N_{\text {stars }}$ & $5 \times 10^{6}$ & $1 \times 10^{5}$ & 400 & $5 \times 10^{9}$ & $1 \times 10^{9}$ & $7 \times 10^{7}$ & $4 \times 10^{12}$ & $3 \times 10^{12}$ & $2 \times 10^{12}$ \\
\hline$Q_{\text {total }}\left[\mathrm{s}^{-1}\right]$ & $9 \times 10^{56}$ & $2 \times 10^{57}$ & $4 \times 10^{62}$ & $2 \times 10^{55}$ & $8 \times 10^{55}$ & $3 \times 10^{56}$ & $1 \times 10^{47}$ & $2 \times 10^{47}$ & $4 \times 10^{47}$ \\
\hline
\end{tabular}

Notes. $M_{\max }$ is the maximum stellar mass permitted by limiting the total star formation rate to $\mathrm{SFR}_{\text {total }}=1 \times 10^{4} M_{\odot} \mathrm{yr}^{-1}$ (no AGN contribution to the FIR emission) and $N_{\text {stars }}$ is the number of stars of this mass.

Table 3. As per Table 2, but for the total star formation rate limited to $\mathrm{SFR}_{\mathrm{total}}=5000 M_{\odot} \mathrm{yr}^{-1}$ (50\% AGN contribution to the FIR emission).

\begin{tabular}{|c|c|c|c|c|c|c|c|c|c|}
\hline \multirow{2}{*}{$\begin{array}{l}M_{\text {total }}\left[M_{\odot}\right] \\
\text { IMF index }\end{array}$} & \multicolumn{3}{|c|}{$4 \times 10^{11}$} & \multicolumn{3}{|c|}{$4 \times 10^{12}$} & \multicolumn{3}{|c|}{$4 \times 10^{13}$} \\
\hline & $\alpha=-2.35$ & $\alpha=-2.60$ & $\alpha=-2.85$ & $\alpha=-2.35$ & $\alpha=-2.60$ & $\alpha=-2.85$ & $\alpha=-2.35$ & $\alpha=-2.60$ & $\alpha=-2.85$ \\
\hline$M_{\max }\left[M_{\odot}\right]$ & 23 & 51 & 190 & 3 & 4 & 6 & 0.7 & 0.6 & 0.6 \\
\hline$N_{\text {stars }}$ & $2 \times 10^{7}$ & $1 \times 10^{6}$ & 8000 & $4 \times 10^{12}$ & $7 \times 10^{9}$ & $1 \times 10^{9}$ & $1 \times 10^{13}$ & $9 \times 10^{12}$ & $8 \times 10^{12}$ \\
\hline$Q_{\text {total }}\left[\mathrm{s}^{-1}\right]$ & $3 \times 10^{56}$ & $4 \times 10^{56}$ & $3 \times 10^{53}$ & $1 \times 10^{54}$ & $1 \times 10^{55}$ & $3 \times 10^{56}$ & $1 \times 10^{43}$ & $2 \times 10^{43}$ & $1 \times 10^{43}$ \\
\hline
\end{tabular}

above the SPIRE confusion limit of $\left.3 \sigma_{\text {conf }}=18 \mathrm{mJy}\right) 2013$ sources for which a modified blackbody could be fit, yielding the dust temperature. Based on these detections we find the following:

- A mean dust temperature of $\left\langle T_{\text {dust }}\right\rangle=31.4 \pm 0.2 \mathrm{~K}$ and a mean spectral emissivity index of $\langle\beta\rangle=0.991 \pm 0.001$;

- No apparent critical ionising photon rate, with $250 \mu \mathrm{m}$ emission being detected up to $Q=8.9 \times 10^{57} \mathrm{~s}^{-1}$. If the $21 \mathrm{~cm}$ results and model are reliable, this suggests that the FIR emission does not exclusively trace the stellar heated dust, implying a significant contribution from an AGN;

- A strong correlation between the dust temperature and the ionising photon rate, which we suspect is driven mainly by the Malmquist bias. Normalising the ionising photon rate by the total FIR luminosity, causes the correlation to disappear. Since the number of luminous AGN is expected to increase with redshift, this may also suggest that the low temperature (FIR) emission can arise from AGN heating of the dust.

By calculating the ionising photon rate expected for each stellar mass, for a total star formation rate of $\mathrm{SFR}_{\mathrm{total}}=1 \times 10^{4} M_{\odot} \mathrm{yr}^{-1}$, the observed ionising photon rates $\left(Q \sim 10^{57} \mathrm{~s}^{-1}\right)$ in the most luminous of the SPIRE sources can be reproduced by several initial mass functions, which give a sufficient population of massive stars. Exceeding the critical value above which all of the neutral atomic gas is believed to be ionised, this raises the question of what fuels the star formation. However, this is very much dictated by the choice of $\mathrm{SFR}_{\text {total }}$ and if $<10^{4} M_{\odot} \mathrm{yr}^{-1}$, due to a large AGN contribution to the FIR luminosity, lower values of $Q_{\text {total }}$ are obtained. In order to address this, a valid IMF for such highly luminous sources is required to determine whether the stellar population can account for the observed ionising photon rates. If this is the case, in the model of Curran \& Whiting (2012) the single large ionising source should be replaced with the putative stellar distribution embedded in the exponential gas disc in order to determine whether such a population could completely ionise the gas.

Acknowledgements. We wish to thank the anonymous referee for the prompt and helpful comments. SWD acknowledges a Victoria Doctoral Scholarship and the support of an Australian Government Research Training Program scholarship administered by Curtin University. This research has made use of Astropy, a community-developed core Python package for Astronomy (Astropy Collaboration 2013), and the NASA/IPAC Extragalactic Database (NED), which is operated by the Jet Propulsion Laboratory, California Institute of Technology, under contract with the National Aeronautics and Space Administration. This research has also made use of NASA's Astrophysics Data System Bibliographic Services.

\section{References}

Aditya, J. N. H. S., \& Kanekar, N. 2018, MNRAS, 473, 59

Aditya, J. N. H. S., Kanekar, N., \& Kurapati, S. 2016, MNRAS, 455, 4000

Aditya, J. N. H. S., Kanekar, N., Prochaska, J. X., et al. 2017, MNRAS, 465, 5011

Alexander, D. M., Bauer, F. E., Brandt, W. N., et al. 2003, AJ, 126, 539

Allison, J. R., Curran, S. J., Emonts, B. H. C., et al. 2012, MNRAS, 423, 2601

Antonucci, R. R. J. 1993, ARA\&A, 31, 473

Astropy Collaboration (Robitaille, T. P., et al.) 2013, A\&A, 558, A33

Barger, A. J., Cowie, L. L., \& Wang, W.-H. 2008, ApJ, 689, 687

Bastian, N., Covey, K. R., \& Meyer, M. R. 2010, ARA\&A, 48, 339

Blain, A. W., Barnard, V. E., \& Chapman, S. C. 2003, MNRAS, 338, 733

Bruzzese, S. M., Meurer, G. R., Lagos, C. D. P., et al. 2015, MNRAS, 447, 618

Calzetti, D., Wu, S.-Y., Hong, S., et al. 2010, ApJ, 714, 1256

Cano-Díaz, M., Maiolino, R., Marconi, A., et al. 2012, A\&A, 537, L8

Casey, C. M. 2012, MNRAS, 425, 3094

Croton, D. J., Springel, V., White, S. D. M., et al. 2006, MNRAS, 365, 11

Curran, S. J., \& Duchesne, S. W. 2018, MNRAS, 476, 3580

Curran, S. J., \& Whiting, M. T. 2010, ApJ, 712, 303

Curran, S. J., \& Whiting, M. T. 2012, ApJ, 759, 117

Curran, S. J., Johansson, L. E. B., Bergman, P., Heikkilä, A., \& Aalto, S. 2001, A\&A, 367, 457

Curran, S. J., Whiting, M. T., Wiklind, T., et al. 2008, MNRAS, 391, 765

Curran, S. J., Whiting, M. T., Murphy, M. T., et al. 2011, MNRAS, 413, 1165

Curran, S., Whiting, M. T., Sadler, E. M., \& Bignell, C. 2013a, MNRAS, 428, 2053

Curran, S. J., Whiting, M. T., Tanna, A., et al. 2013b, MNRAS, 429, 3402

Curran, S., Allison, J. R., Whiting, M. T., et al. 2016, MNRAS, 457, 3666

Curran, S., Whiting, M. T., Allison, J. R., et al. 2017a, MNRAS, 467, 4514

Curran, S. J., Hunstead, R. W., Johnston, H. M., et al. 2017b, MNRAS, 470, 4600

Curran, S. J., Hunstead, R. W., Johnston, H. M., et al. 2019, MNRAS, 484, 1182

Dale, D. A., \& Helou, G. 2002, ApJ, 576, 159

de Grijp, M. H. K., Miley, G. K., Lub, J., \& de Jong, T. 1985, Nature, 314, 240

Di Matteo, T., Springel, V., \& Hernquist, L. 2005, Nature, 433, 604

Diolaiti, E., Bendinelli, O., Bonaccini, D., et al. 2000, in Adaptive Optical Systems Technology, Proc. SPIE, 4007, 879 
S. J. Curran and S. W. Duchesne: Ultraviolet photo-ionisation in far-infrared selected sources

Elvis, M., Civano, F., Vignali, C., et al. 2009, ApJS, 184, 158

Fabian, A. C. 1999, MNRAS, 308, L39

Fabian, A. C. 2012, ARA\&A, 50, 455

Farrah, D., Urrutia, T., Lacy, M., et al. 2012, ApJ, 745, 178

Galametz, M., Kennicutt, R. C., Albrecht, M., et al. 2012, MNRAS, 425, 763

Galliano, F., Galametz, M., \& Jones, A. P. 2018, ARA\&A, 56, 673

Geréb, K., Maccagni, F. M., Morganti, R., \& Oosterloo, T. A. 2015, A\&A, 575 44

Grasha, K., \& Darling, J. 2011, Am. Astron. Soc. Meeting Abstracts, 43, 345.02

Grasha, K., Darling, J.K., Bolatto, A.D., Leroy, A., \& Stocke, J. 2019, ApJ, submitted

Groves, B., Krause, O., Sandstrom, K., et al. 2012, MNRAS, 426, 892

Hardcastle, M. J., Evans, D. A., \& Croston, J. H. 2007, MNRAS, 376, 1849

Harrison, C. M., Alexander, D. M., Mullaney, J. R., et al. 2012, ApJ, 760, L15

Hatziminaoglou, E., Omont, A., Stevens, J. A., et al. 2010, A\&A, 518, L33

Heckman, T. M., \& Best, P. N. 2014, ARA\&A, 52, 589

Hildebrand, R. H. 1983, Q. J1 R. ast. Soc., 24, 267

Kalberla, P. M. W., \& Kerp, J. 2009, ARA\&A, 47, 27

Kennicutt, Jr., R. C. 1998, ApJ, 498, 541

Kovács, A., Omont, A., Beelen, A., et al. 2010, ApJ, 717, 29

Kroupa, P. 2001, MNRAS, 322, 231

Li, Y., Crocker, A. F., Calzetti, D., et al. 2013, ApJ, 768, 180

Magdis, G. E., Rigopoulou, D., Helou, G., et al. 2013, A\&A, 558, A136

Massey, P. 2002, ApJS, 141, 81

Morić, I., Smolčić, V., Kimball, A., et al. 2010, ApJ, 724, 779
Nardini, E., Risaliti, G., Watabe, Y., Salvati, M., \& Sani, E. 2010, MNRAS, 405, 2505

Nguyen, H. T., Schulz, B., Levenson, L., et al. 2010, A\&A, 518, L5

Oliver, S. J., Bock, J., Altieri, B., et al. 2012, MNRAS, 424, 1614

Osterbrock, D. E. 1989, Astrophysics of Gaseous Nebulae and Active Galactic Nuclei (Mill Valley, California: University Science Books)

Page, M. J., Symeonidis, M., Vieira, J. D., et al. 2012, Nature, 485, 213

Rieke, G., \& Lebofsky, M. 1979, ARA\&A, 17, 477

Roos, O., Juneau, S., Bournaud, F., \& Gabor, J. M. 2015, ApJ, 800, 19

Roseboom, I. G., Oliver, S. J., Kunz, M., et al. 2010, MNRAS, 409, 48

Roseboom, I. G., Ivison, R. J., Greve, T. R., et al. 2012, MNRAS, 419, 2758

Salpeter, E. E. 1955, ApJ, 121, 161

Schlegel, D. J., Finkbeiner, D. P., \& Davis, M. 1998, ApJ, 500, 525

Silk, J., \& Rees, M. J. 1998, A\&A, 331, L1

Skrutskie, M. F., Cutri, R. M., Stiening, R., et al. 2006, AJ, 131, 1163

Smith, A. J., Wang, L., Oliver, S. J., et al. 2012, MNRAS, 419, 377

Trouille, L., Barger, A. J., Cowie, L. L., Yang, Y., \& Mushotzky, R. F. 2008, ApJS, 179, 1

Úbeda, L., Maíz-Apellániz, J., \& MacKenty, J. W. 2007, AJ, 133, 932

Urry, C. M., \& Padovani, P. 1995, PASP, 107, 803

Weisz, D. R., Johnson, L. C., Foreman-Mackey, D., et al. 2015, ApJ, 806, 198

Wright, E. L., Eisenhardt, P. R. M., Mainzer, A. K., et al. 2010, AJ, 140, 1868

Xue, Y. Q., Luo, B., Brandt, W. N., et al. 2011, ApJS, 195, 10

Yang, M., Greve, T. R., Dowell, C. D., \& Borys, C. 2007, ApJ, 660, 1198

Younger, J. D., Omont, A., Fiolet, N., et al. 2009, MNRAS, 394, 1685 


\section{Appendix A: UV photo-ionisation of the Chandra Deep Field North sources}

The original aim of the project was to test the Page et al. (2012) sample for a similar absence of $250 \mu \mathrm{m}$ emission above the critical UV luminosity where $\mathrm{H}_{\mathrm{I}} 21 \mathrm{~cm}$ absorption is not detected. As an initial step we reproduced the method of Page et al. (2012), cross-matching the Chandra Deep Field North (CDF-N, Alexander et al. 2003) with spectroscopic redshifts, obtained from Barger et al. (2008) and Trouille et al. (2008) and from the references listed in Table S2 in Page et al. (2012). For each source for which we could obtain a redshift, we calculated the $k$-corrected X-ray luminosity, $L_{\mathrm{X}}$, from the flux density, assuming $S_{v} \propto v^{-0.9}$. We then cross-matched them with the sources within 6 arcsec of those searched by HerMES. These matches are unique (i.e. no sources within SPIRE are matched to multiple CDF-N AGN).

In Fig. A. 1 we show the resulting $2-8 \mathrm{keV}$ X-ray luminosity versus redshift for the $250 \mu \mathrm{m}$ searched sources. This is identical to the distribution of Page et al. (2012), above the $L_{X}<$ $10^{42} \mathrm{erg} \mathrm{s}^{-1}\left(<10^{35} \mathrm{~W}\right)$ cut, apart from an additional source at $z=1.3709$ (source number 40 in Trouille et al. 2008), which has $L_{\mathrm{X}}=1.6 \times 10^{37} \mathrm{~W}$ and $S_{\mathrm{FIR}}=40.1 \mathrm{mJy}^{5}$. Examining the statistics, below the $L_{\mathrm{X}}=10^{37} \mathrm{~W}$ cut-off, there are $10 \mathrm{FIR}$ detections and 34 non-detections, giving a detection probability of $p=$ 0.227 . Applying this to the $L_{\mathrm{X}}>10^{37} \mathrm{~W}$ sample, the binomial probability of obtaining zero $250 \mu \mathrm{m}$ detections in 21 sources is $P($ bin $)=4.485 \times 10^{-3}$. This is significant at $S($ bin $)=2.84 \sigma$ and consistent with Page et al. (2012) finding a $>99 \%(>2.58 \sigma)$ significance from a single-tail Fisher's exact test. This significance does decrease, however, when source number 40 is included or when the whole sample is tested (Table A.1). Thus, the absence of $250 \mu \mathrm{m}$ emission in luminous X-ray sources is not particularly significant, especially compared to $S$ (bin) $=5.66 \sigma$ for the absence of $21 \mathrm{~cm}$ absorption in the UV luminous sources (Curran et al. 2019).

Following the SED fitting procedure described in Sect. 2.2, there is sufficient photometry to obtain the ionising photon rate for 103 of the CDF-N sources (Fig. A.2). The highest ionising photon rate at which $250 \mu \mathrm{m}$ is detected is $Q=4.4 \times 10^{55} \mathrm{~s}^{-1}$, which is consistent with the value above which $\mathrm{H}_{\mathrm{I}} 21 \mathrm{~cm}$ absorption remains undetected (Sect. 1). However, the $p=0.134$ detection rate below this gives a binomial probability of $P($ bin $)=$ 0.137 for zero detections out of the 14 sources with $Q>4.4 \times$ $10^{55} \mathrm{~s}^{-1}$, which is only significant at $S$ (bin) $=1.51 \sigma$. Moreover, when tested over a larger sample no critical rate is apparent (Sect. 3.1). Furthermore, extending the Page et al. (2012) sam-

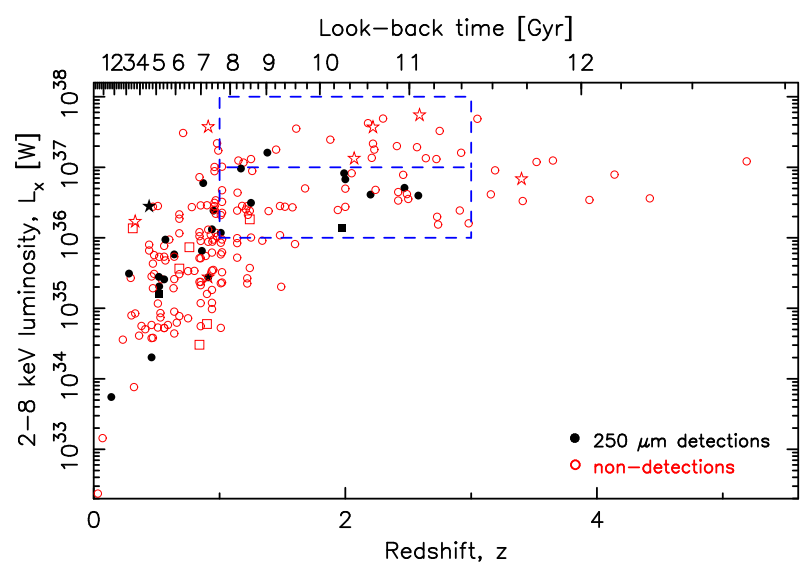

Fig. A.1. 2-8 keV X-ray luminosity vs. redshift for the sources in the CDF-N searched in $250 \mu \mathrm{m}$ by SPIRE. The dashed lines enclose the $L_{\mathrm{X}}=10^{43}-10^{44} \mathrm{erg} \mathrm{s}^{-1}$ and $L_{\mathrm{X}}=10^{44}-10^{45} \mathrm{erg} \mathrm{s}^{-1}$ regions spanning $z=1-3$ (cf. Fig. 1 in Page et al. 2012). The filled symbols show those detected in $250 \mu \mathrm{m}$ emission $\left(S_{\mathrm{FIR}}>18 \mathrm{mJy}\right)$, and unfilled for undetected, with the shapes representing the NED classification: stars for QSOs, circles for galaxies, and squares for unclassified.

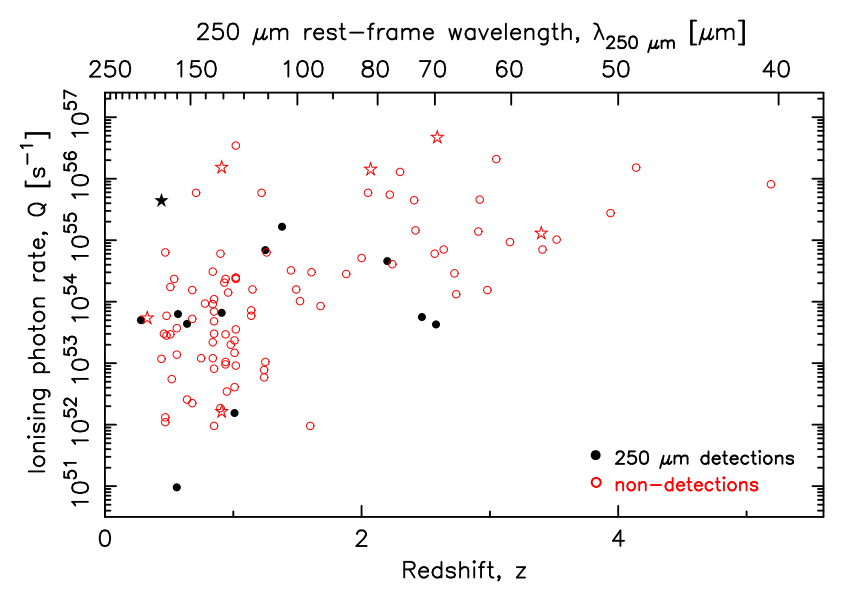

Fig. A.2. Ionising photon rate vs. redshift for the sources in the CDF-N. The symbols are as per Fig. A.1.

ple, using the Chandra Deep Field South (CDF-S, Xue et al. 2011) and COSMOS fields (Elvis et al. 2009), finds no evidence for suppressed star formation at high X-ray luminosities (Harrison et al. 2012).

Table A.1. $250 \mu \mathrm{m}$ detection statistics above and below $L_{\mathrm{X}}=10^{44} \mathrm{erg} \mathrm{s}^{-1}\left(10^{37} \mathrm{~W}\right)$ for the $L_{\mathrm{X}}=10^{37}-10^{39} \mathrm{~W}-z=1-3$ sample, the $L_{\mathrm{X}}>10^{35} \mathrm{~W}$ $\left(>10^{42} \mathrm{erg} \mathrm{s}^{-1}\right.$ ) sample, and the whole sample (Page et al. 2012), in addition to the effect on each by including source number 40.

\begin{tabular}{|c|c|c|c|c|c|c|c|c|c|}
\hline \multirow[t]{2}{*}{ Sample } & \multicolumn{2}{|c|}{$L_{\mathrm{X}}<10^{37} \mathrm{~W}$} & \multirow[b]{2}{*}{$p$} & \multirow{2}{*}{$\begin{array}{l}\text { Source } \\
\text { No. } 40\end{array}$} & \multirow[t]{2}{*}{$n_{\text {total }}$} & \multicolumn{2}{|c|}{$L_{\mathrm{X}} \geq 10^{37} \mathrm{~W}$} & \multirow[t]{2}{*}{$P($ bin $)$} & \multirow[t]{2}{*}{$S$ (bin) } \\
\hline & Non-detections & Detections & & & & Non-detections & Detections & & \\
\hline \multirow[t]{2}{*}{$L_{\mathrm{X}}=10^{36}-10^{38} \mathrm{~W}$} & 34 & 10 & 0.227 & Yes & 66 & 21 & 1 & 0.0257 & $2.23 \sigma$ \\
\hline & & & & No & 65 & 21 & 0 & $4.485 \times 10^{-3}$ & $2.84 \sigma$ \\
\hline \multirow[t]{2}{*}{$L_{\mathrm{X}}>10^{35} \mathrm{~W}$} & 122 & 23 & 0.159 & Yes & 176 & 30 & 1 & 0.0325 & $2.14 \sigma$ \\
\hline & & & & No & 175 & 30 & 0 & $5.620 \times 10^{-3}$ & $2.77 \sigma$ \\
\hline \multirow[t]{2}{*}{ Whole sample } & 149 & 24 & 0.139 & Yes & 205 & 30 & 1 & 0.0585 & $1.89 \sigma$ \\
\hline & & & & No & 204 & 30 & 0 & 0.0113 & $2.53 \sigma$ \\
\hline
\end{tabular}

Notes. $p$ gives the probability of a detection based upon the detection rate at $L_{\mathrm{X}}<10^{37} \mathrm{~W}$; the last two columns give the binomial probability and significance of this observed number of $250 \mu \mathrm{m}$ detections or fewer at $L_{\mathrm{X}}>10^{37} \mathrm{~W}$.

\footnotetext{
5 This source, SDSS J123553.13+621037.3, has a 0.64 arcsec offset from the optical and a 5.9 arcsec offset from the $250 \mu \mathrm{m}$ source, and so just falls within the 6 arcsec search radius.
} 